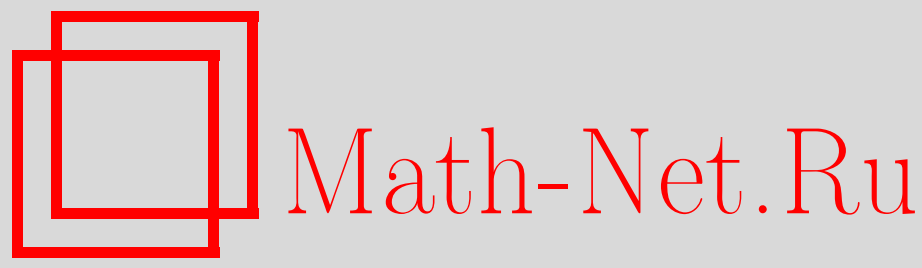

А. М. Синев, Квантовые траектории осциллятора, взаимодействующего с электромагнитным полем, классической силой и термостатом, ТМФ, 2009, том 158, номер 3, 444-459

DOI: https://doi.org/10.4213/tmf6326

Использование Общероссийского математического портала Math-Net.Ru подразумевает, что вы прочитали и согласны с пользовательским соглашением http: //www . mathnet.ru/rus/agreement

Параметры загрузки:

IP : 52.6 .47 .48

26 апреля 2023 г., 14:52:30

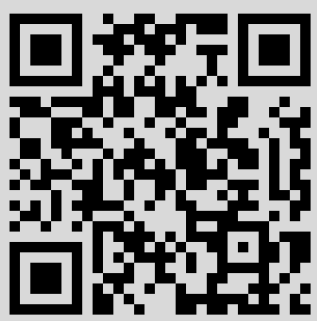




\title{
КВАНТОВЫЕ ТРАЕКТОРИИ ОСЦИЛЛЯТОРА, ВЗАИМОДЕЙСТВУЮЩЕГО С ЭЛЕКТРОМАГНИТНЫМ ПОЛЕМ, КЛАССИЧЕСКОЙ СИЛОЙ И ТЕРМОСТАТОМ
}

\begin{abstract}
Рассмотрена точно решаемая задача об описании динамики квантового осциллятора, взаимодействующего с электромагнитным полем, классической силой и термостатом. Предложен общий метод решения уравнений марковской эволюции системы - метод квантовых траекторий. Построение стохастического оператора эволюции включает в себя вывод стохастического аналога формулы Бейкера-Хаусдорфа. Вычислена матрица плотности системы для произвольного начального состояния. В качестве физического приложения рассчитано влияние окружения при ненулевой температуре на точность измерений слабой классической силы интерференционным методом.
\end{abstract}

Ключевые слова: открытые квантовые системы, стохастические дифференциальные уравнения, квантовые измерения.

\section{1. ВВЕДЕНИЕ}

При исследовании поведения квантовой системы с небольшим числом степеней свободы, взаимодействующей с окружением (системой большого числа степеней свободы), данная система понимается как открытая. На унитарную эволюцию системы накладывается квантовый шум от воздействия на нее окружения [1]. Таким образом, динамика открытой системы описывается с помощью либо квантовых уравнений Ланжевена и квантовых стохастических дифференциальных уравнений, либо управляющих уравнений для оператора плотности, что соответствует представлению Шредингера. Физические примеры эволюции открытых квантовых систем можно найти в работах [1], [2].

Концепция открытой квантовой системы широко применяется в теории квантовых измерений, где окружением является классический измерительный прибор, с которого считывается информация о системе. В этом случае важными оказываются так называемые апостериорные состояния, описываемые стохастическим уравнением Шредингера, которое рассмотрено в работах [3], [4].

* Московский государственный технический университет им. Н.Э. Баумана, Москва, Россия. E-mail: sansin@mail.ru 
Среди неоператорных методов описания эволюции открытых квантовых систем отметим метод ограниченных интегралов по путям Менского (обобщение континуального интеграла Фейнмана) [5] и теорию квантовой томографии, которая основана на описании состояния системы с помощью функции Вигнера и с которой можно ознакомиться в работах [6], [7].

В настоящей работе мы рассматриваем модельное точно решаемое уравнение марковской эволюции, применяя метод квантовых траекторий [8], позволяющий представить решение задачи Коши

$$
\frac{d \rho_{t}}{d t}=\mathcal{L}\left(\rho_{t}\right),\left.\quad \rho\right|_{t=0}=\rho_{0},
$$

где $\mathcal{L}(\cdot)$ - генератор вполне положительной полугруппы $\mathcal{T}_{t}(\cdot): \rho_{t}=\mathcal{T}_{t}\left(\rho_{0}\right)$, сохраняющей след, в виде математического ожидания

$$
\rho_{t}=\mathrm{M} u_{t} \rho_{0} u_{t}^{*}
$$

где $\rho_{0}$ - начальное состояние системы (положительный оператор с единичным следом), $u_{t}$ - стохастический оператор эволюции состояния системы (квантовая траектория), удовлетворяющий стохастическому дифференциальному уравнению Ито, сопоставляемому генератору $\mathcal{L}$ марковской эволюции таким образом, чтобы состояние квантовой системы $\rho_{t}$ описывалось уравнением Линдблада

$$
\begin{aligned}
d \rho_{t} & =\mathrm{M} d u_{t} \rho_{0} u_{t}^{*}+\mathrm{M} u_{t} \rho_{0} d u_{t}^{*}+\mathrm{M} d u_{t} \rho_{0} d u_{t}^{*}=\mathcal{L}\left(\rho_{t}\right) d t, \\
d u_{t} & =\left(A d t+B d w_{t}\right) u_{t},
\end{aligned}
$$

где $A, B=\left\{B_{k}\right\}$ - операторные коэффициенты, описывающие обратимые и необратимые процессы взаимодействия квантовой системы и ее окружения, $w_{t}=\left\{w_{t}^{(k)}\right\}-$ независимые стандартные винеровские процессы.

В разделе 2 рассматривается задача Коши для уравнения на матрицу плотности системы, представляющую собой квантовый одномерный гармонический осциллятор, взаимодействующий при ненулевой температуре с окружением, классической силой и с давлением света. Мы находим точное выражение для $u_{t}$, стохастическая часть которого удовлетворяет уравнению первого порядка относительно некоммутирующих операторов

$$
d u_{t}^{\text {stoch }}=\left(f_{t} d X_{t}+g_{t} d Y_{t}+\alpha_{t} b d X_{t}+\beta_{t} b^{\dagger} d Y_{t}\right) u_{t}^{\text {stoch }}
$$

где $f_{t}, g_{t}, \alpha_{t}, \beta_{t}$ - заданные комплексные функции времени, $b^{\dagger}$ и $b$ - операторы рождения и уничтожения с коммутатором $\left[b, b^{\dagger}\right]=I, X_{t}, Y_{t}$ - независимые стандартные винеровские процессы:

$$
\mathrm{M} d X_{t}=\mathrm{M} d Y_{t}=\mathrm{M} d X_{t} d Y_{t}=0, \quad \mathrm{M} d X_{t}^{2}=\mathrm{M} d Y_{t}^{2}=d t .
$$

В разделе 3 вычисляется $\rho_{t}$ для произвольного начального состояния, т.е. строится явный вид полугруппы $\mathcal{T}_{t}(\cdot)$. В разделе 4 вычисляется предельная эволюция оператора плотности осциллятора при $t \rightarrow \infty$. В разделе 5 рассматриваются интерференционный метод измерения классической силы и влияние ненулевой температуры окружения на точность измерений. 


\section{2. ГАМИЛЬТОНИАН СИСТЕМЫ И СТОХАСТИЧЕСКИЙ ОПЕРАТОР ЭВОЛЮЦИИ}

Рассмотрим в пространстве $l_{2}$ генератор линдбладовского типа [9]

$$
\mathcal{L}(\rho)=\frac{\lambda(\nu+1)}{2}\left(2 b \rho b^{\dagger}-b^{\dagger} b \rho-\rho b^{\dagger} b\right)+\frac{\lambda \nu}{2}\left(2 b^{\dagger} \rho b-b b^{\dagger} \rho-\rho b b^{\dagger}\right),
$$

где $b^{\dagger}$ и $b$ - операторы рождения и уничтожения с коммутатором $\left[b, b^{\dagger}\right]=I$. Состояние Гиббса

$$
\rho_{\nu}=\left(1-c_{\nu}\right) \sum_{n} c_{\nu}^{n}|n\rangle\langle n|, \quad c_{\nu}=\frac{\nu}{\nu+1},
$$

является стационарным состоянием данного генератора, т.е. $\mathcal{L}\left(\rho_{\nu}\right)=0$, так как для любого $n$ константа $c=c_{\nu}$ удовлетворяет уравнению

$$
(\nu+1)\{(n+1) c-n\}+\nu\left\{c^{-1} n-(n+1)\right\}=0 .
$$

Генератор (3), известный в квантовой оптике (см., например, [2]), описывает взаимодействие системы с окружением при ненулевой температуре, где $\lambda>0$ характеризует интенсивность обмена энергией между системой и термостатом, а $\nu>0$ выражается через его температуру $T$. Для одномерного осциллятора с частотой $\Omega$ температура $T$ связана с параметром $\nu$ соотношением $\hbar \Omega /(k T)=\ln ((\nu+1) / \nu)$.

Системой, рассматриваемой в настоящей статье, является когерентное лазерное излучение с угловой частотой $\omega$, заполняющее полость между закрепленным зеркалом и подвижным, которое описывается как одномерный квантовый осциллятор с собственной частотой $\Omega$. Данная полость является частью интерферометра, позволяющего измерить малые перемещения подвижного зеркала при воздействии на него слабой классической силы. Гамильтониан системы "излучение-осциллятор" в пространстве $l_{2} \otimes l_{2}$ имеет вид [10], [11]

$$
H_{t}=\hbar\left[\omega a^{\dagger} a \otimes I+I \otimes \Omega b^{\dagger} b+\left(g a^{\dagger} a+f_{t}\right) \otimes\left(b^{\dagger}+b\right)\right],
$$

где первые два слагаемых - собственная энергия лазерного излучения и осциллятора, $a^{\dagger}, a, b^{\dagger}, b$ - операторы рождения и уничтожения в пространстве состояний излучения и осциллятора соответственно, а последнее слагаемое - энергия взаимодействия осциллятора с излучением (давление электромагнитного поля), где $g=-(\omega / L) \sqrt{\hbar /(2 m \Omega)}, m$ - масса осциллятора, $L$ - расстояние между зеркалами, и с внешней силой $f_{t}=-F(t) / \sqrt{2 m \Omega \hbar}$, где $F(t)$ - классическая сила.

Подвижное зеркало через подвес спонтанно обменивается энергией с окружением при ненулевой температуре. При этом состояние $\rho_{t}$ системы определяется квантовым кинетическим уравнением

$$
\begin{gathered}
\frac{d \rho_{t}}{d t}=\frac{i}{\hbar}\left[\rho_{t}, H_{t}\right]+\frac{\lambda(\nu+1)}{2}\left(2 b \rho_{t} b^{\dagger}-b^{\dagger} b \rho_{t}-\rho_{t} b^{\dagger} b\right)+ \\
+\frac{\lambda \nu}{2}\left(2 b^{\dagger} \rho_{t} b-b b^{\dagger} \rho_{t}-\rho_{t} b b^{\dagger}\right)
\end{gathered}
$$

с гамильтонианом (4) и заданным начальным состоянием $\rho_{0}$. 
Будем искать решение уравнения (5) в виде $\rho_{t}=\mathrm{M} u_{t} \rho_{0} u_{t}^{*}$, где $u_{t}$ - стохастический оператор эволюции, удовлетворяющий уравнению

$$
\begin{aligned}
d u_{t}= & {\left[-i \omega a^{\dagger} a d t-i \Omega b^{\dagger} b d t-i\left(g a^{\dagger} a+f_{t}\right)\left(b^{\dagger}+b\right) d t-\right.} \\
& \left.\quad-\frac{\lambda(\nu+1)}{2} b^{\dagger} b d t-\frac{\lambda \nu}{2} b b^{\dagger} d t+\sqrt{\lambda(\nu+1)} b d X_{t}+\sqrt{\lambda \nu} b^{\dagger} d Y_{t}\right] u_{t}
\end{aligned}
$$

с $u_{0}=I$. Здесь $X_{t}, Y_{t}$ - независимые стандартные винеровские процессы. Формула (5) является следствием уравнения (6) и классической формулы дифференцирования Ито. Следует отметить, что операторнозначные коэффициенты в уравнении (6), действующие в пространстве состояний осциллятора, не коммутируют в различные моменты времени. Распутывая операторы при дифференциале $d t$ по формуле Бейкера-Хаусдорфа [8], выделим стохастическую часть $\varphi_{t}$ уравнения (6):

$$
\begin{gathered}
\varphi_{t} \stackrel{\text { def }}{=} e^{\lambda \nu t / 2} e^{i \omega a^{\dagger} a t} e^{C_{t}} e^{i \beta_{t}^{-} b} e^{i \beta_{t}^{+} b^{\dagger}} e^{b^{\dagger} b(\lambda(2 \nu+1) / 2+i \Omega) t} u_{t} \\
d \varphi_{t}=\left[-i q_{t} \beta_{t}^{+} d X_{t}+i \sigma_{t} \beta_{t}^{-} d Y_{t}+q_{t} b d X_{t}+\sigma_{t} b^{\dagger} d Y_{t}\right] \varphi_{t}, \quad \varphi_{0}=I \\
q_{t}=\sqrt{\lambda(\nu+1)} \exp \left[-\frac{\lambda}{2}(2 \nu+1) t-i \Omega t\right], \quad \sigma_{t}=\sqrt{\lambda \nu} \exp \left[\frac{\lambda}{2}(2 \nu+1) t+i \Omega t\right] \\
\beta_{t}^{ \pm}=\int_{0}^{t} A_{\tau} \exp \left[ \pm \frac{\lambda}{2}(2 \nu+1) \pm i \Omega \tau\right] d \tau \\
C_{t}=\int_{0}^{t} A_{\tau} \exp \left[-\frac{\lambda}{2}(2 \nu+1) \tau-i \Omega \tau\right] \beta_{\tau}^{+} d \tau
\end{gathered}
$$

где $A_{t}=g a^{\dagger} a+f_{t}$.

Решение уравнения (8) использует стохастический аналог формулы Бейкера-Хaусдорфа. Поскольку уравнение (8) является стохастическим уравнением первого порядка относительно операторов $b$ и $b^{\dagger}$, его решение имеет вид композиции экспонент от операторов второго порядка по $b$ и $b^{\dagger}$ :

$$
\begin{aligned}
& \varphi_{t}=E_{1} E_{2} E_{3} E_{4} \\
& E_{1}=\exp \left[\int_{0}^{t} s_{\tau} d X_{\tau}+\int_{0}^{t} i \sigma_{\tau} \beta_{\tau}^{-} d Y_{\tau}-\frac{1}{2} \int_{0}^{t}\left(s_{\tau}^{2}-\sigma_{\tau}^{2} \beta_{\tau}^{-2}\right) d \tau\right] \\
& E_{2}=\exp \left[b^{\dagger} \int_{0}^{t}\left(\mu_{\tau} d X_{\tau}+\sigma_{\tau} d Y_{\tau}+k_{\tau} d \tau\right)-\frac{\left(b^{\dagger}\right)^{2}}{2} \int_{0}^{t}\left(\mu_{\tau}^{2}+\sigma_{\tau}^{2}\right) d \tau\right] \\
& E_{3}=\exp \left[b^{\dagger} b \int_{0}^{t} \psi_{\tau} d \tau\right] \\
& E_{4}=\exp \left[b \int_{0}^{t}\left(\eta_{\tau} d X_{\tau}+h_{\tau} d \tau\right)-\frac{b^{2}}{2} \int_{0}^{t} \eta_{\tau}^{2} d \tau\right]
\end{aligned}
$$


Принимая во внимание многомерную формулу Ито (см., например, [12]) и коммутационные соотношения

$$
e^{\alpha b^{\dagger}+\beta\left(b^{\dagger}\right)^{2}} b=\left(b-\alpha-2 \beta b^{\dagger}\right) e^{\alpha b^{\dagger}+\beta\left(b^{\dagger}\right)^{2}}, \quad e^{\alpha b^{\dagger} b} b=b e^{-\alpha} e^{\alpha b^{\dagger} b},
$$

приравниваем коэффициенты в левой и правой частях уравнения (8) при соответствующих степенях операторов $b, b^{\dagger}$ и получаем систему уравнений для нахождения неизвестных функций $s_{t}, \mu_{t}, k_{t}, \psi_{t}, \eta_{t}, h_{t}$

$$
\begin{gathered}
\mu_{t}=-q_{t} \int_{0}^{t}\left(\mu_{\tau}^{2}+\sigma_{\tau}^{2}\right) d \tau \\
\eta_{t}=q_{t} \exp \left[-\int_{0}^{t} q_{\tau} \mu_{\tau} d \tau\right], \quad \psi_{t}=-q_{t} \mu_{t} \\
h_{t}=-\eta_{t} s_{t}, \quad k_{t}=-\mu_{t} s_{t}-i \sigma_{t}^{2} \beta_{t}^{-}, \\
s_{t}=-i q_{t} \beta_{t}^{+}+q_{t} \int_{0}^{t}\left(\mu_{\tau} d X_{\tau}+\sigma_{\tau} d Y_{\tau}+k_{\tau} d \tau\right) .
\end{gathered}
$$

Функция $\mu_{t}$ удовлетворяет уравнению Риккати (13). После того, как найдено $\mu_{t}$, определяются остальные функции. Для удобства введем одномерные диффузионные процессы в показателях экспонент (11), (12)

$$
z_{t}=\int_{0}^{t}\left(\mu_{\tau} d X_{\tau}+\sigma_{\tau} d Y_{\tau}+k_{\tau} d \tau\right), \quad w_{t}=\int_{0}^{t}\left(\eta_{\tau} d X_{\tau}+h_{\tau} d \tau\right)
$$

Тогда из $(15),(16)$ следует, что $s_{t}=-i q_{t} \beta_{t}^{+}+q_{t} z_{t}$, а процессы $z_{t}$ и $w_{t}$ удовлетворяют системе линейных стохастических дифференциальных уравнений

$$
\begin{array}{ll}
d z_{t}=-\left(q_{t} \mu_{t}\left(z_{t}-i \beta_{t}^{+}\right)+i \sigma_{t}^{2} \beta_{t}^{-}\right) d t+\mu_{t} d X_{t}+\sigma_{t} d Y_{t}, & z_{0}=0 \\
d w_{t}=-q_{t} \eta_{t}\left(z_{t}-i \beta_{t}^{+}\right) d t+\eta_{t} d X_{t}, & w_{0}=0 .
\end{array}
$$

Таким образом, решение уравнения (8) имеет вид

$$
\begin{aligned}
\varphi_{t}= & \exp \left[\int_{0}^{t} q_{\tau}\left(z_{\tau}-i \beta_{\tau}^{+}\right) d X_{\tau}\right] \exp \left[i \int_{0}^{t} \sigma_{\tau} \beta_{\tau}^{-} d Y_{\tau}\right] \times \\
& \times \exp \left[-\frac{1}{2} \int_{0}^{t}\left(q_{\tau}^{2}\left(z_{\tau}-i \beta_{\tau}^{+}\right)^{2}-\sigma_{\tau}^{2}\left(\beta_{\tau}^{-}\right)^{2}\right) d \tau\right] \times \\
& \times \exp \left[b^{\dagger} z_{t}-\frac{\left(b^{\dagger}\right)^{2}}{2} \int_{0}^{t}\left(\mu_{\tau}^{2}+\sigma_{\tau}^{2}\right) d \tau\right] \times \\
& \times \exp \left[-b^{\dagger} b \int_{0}^{t} q_{\tau} \mu_{\tau} d \tau\right] \exp \left[b w_{t}-\frac{b^{2}}{2} \int_{0}^{t} \eta_{\tau}^{2} d \tau\right]
\end{aligned}
$$


Учитывая (7), окончательно запишем решение уравнения (6) в виде композиции экспонент от операторов $b$ и $b^{\dagger}$ :

$$
\begin{aligned}
u_{t}= & e^{-\lambda \nu t / 2} \exp \left[\int_{0}^{t} q_{\tau}\left(z_{\tau}-i \beta_{\tau}^{+}\right) d X_{\tau}\right] \exp \left[i \int_{0}^{t} \sigma_{\tau} \beta_{\tau}^{-} d Y_{\tau}\right] \times \\
& \times \exp \left[-\frac{1}{2} \int_{0}^{t}\left(q_{\tau}^{2}\left(z_{\tau}-i \beta_{\tau}^{+}\right)^{2}-\sigma_{\tau}^{2}\left(\beta_{\tau}^{-}\right)^{2}\right) d \tau\right] \times \\
& \times e^{-i \omega a^{\dagger} a t} e^{-C_{t}} e^{-b^{\dagger} b(\lambda(2 \nu+1) / 2+i \Omega) t} e^{-i \beta_{t}^{+} b^{\dagger}} e^{-i \beta_{t}^{-} b} \times \\
& \times \exp \left[b^{\dagger} z_{t}-\frac{\left(b^{\dagger}\right)^{2}}{2} \int_{0}^{\tau}\left(\mu_{\tau}^{2}+\sigma_{\tau}^{2}\right) d \tau\right] \times \\
& \times \exp \left[-b^{\dagger} b \int_{0}^{t} q_{\tau} \mu_{\tau} d \tau\right] \exp \left[b w_{t}-\frac{b^{2}}{2} \int_{0}^{t} \eta_{\tau}^{2} d \tau\right] .
\end{aligned}
$$

\section{3. СОСТОЯНИЕ СИСТЕМЫ В ПРОИЗВОЛЬНЫЙ МОМЕНТ ВРЕМЕНИ}

Построим решение уравнения (5) $\rho_{t}=\mathrm{M} u_{t} \rho_{0} u_{t}^{*}$, где $u_{t}$ определена в формуле (20), не конкретизируя начальное состояние $\rho_{0}$. Вычисление данного математического ожидания не является тривиальной задачей, так как процесс $\int_{0}^{t} q_{\tau} z_{\tau} d X_{\tau}$, входящий в $u_{t}(20)$, не гауссовский.

Рассмотрим в пространстве состояний осциллятора нормальный символ $\phi_{t}(\bar{g}, g$, $\bar{h}, h)$ супероператора $\varphi_{t}(\cdot) \varphi_{t}^{*}$, действующего на $\rho_{0}=|h\rangle\left\langle\left. h\right|_{\text {osc }} \otimes \rho_{0}^{\text {las }}\right.$, где $\left.\mid h\right\rangle=$ $e^{-|h|^{2} / 2}\left(1, h, h^{2} / \sqrt{2 !}, \ldots, h^{n} / \sqrt{n !}, \ldots\right)$ - нормированный когерентный вектор из пространства состояний осциллятора $l_{2}, h \in \mathbb{C}, h \neq 0$, с функцией $\varphi_{t}$, определенной в (19). Кроме того, операторнозначные функции $z_{t}, w_{t}$ и $\beta_{t}^{ \pm}$, действующие в пространстве состояний лазерного излучения, коммутируют друг с другом в соответствии с (10), (18). Их расположение относительно $\rho_{0}^{\text {las }}$ отметим стрелкой, например $\overrightarrow{z_{t}}\left(\overleftarrow{z_{t}}\right)$ означает, что $z_{t}$ действует слева (справа) на $\rho_{0}^{\text {las }}$. Таким образом, имеем

$$
\begin{aligned}
& \phi_{t} \stackrel{\text { def }}{=} e^{|g|^{2}} e^{|h|^{2}}\left\langleg \left|\varphi _ { t } \left(|h\rangle\left\langle\left. h\right|_{\text {osc }} \otimes \rho_{0}^{\text {las }}\right) \varphi_{t}^{*}|g\rangle=\exp \left[\int_{0}^{t}\left[q_{\tau}\left(\vec{z}_{\tau}-i \vec{\beta}_{\tau}^{+}\right)+\text {э.c. }\right] d X_{\tau}\right] \times\right.\right.\right. \\
& \times \exp \left[\int_{0}^{t}\left[i \sigma_{\tau} \vec{\beta}_{\tau}^{-}+\text {э.c. }\right] d Y_{\tau}\right] \times \\
& \times \exp \left[-\frac{1}{2} \int_{0}^{t}\left[q_{\tau}^{2}\left(\vec{z}_{\tau}-i \vec{\beta}_{\tau}^{+}\right)^{2}-\sigma_{\tau}^{2}\left(\vec{\beta}_{\tau}^{-}\right)^{2}+\text { э.c. }\right] d \tau\right] \times \\
& \times \exp \left[\bar{g} \vec{z}_{t}-\frac{\bar{g}^{2}}{2} \int_{0}^{t}\left(\mu_{\tau}^{2}+\sigma_{\tau}^{2}\right) d \tau+\text { э.c. }\right] \exp \left[\bar{g} h\left(e^{-\int_{0}^{t} q_{\tau} \mu_{\tau} d \tau}-1\right)+\text { э.c. }\right] \times \\
& \times \exp \left[h \vec{w}_{t}-\frac{h^{2}}{2} \int_{0}^{t} \eta_{\tau}^{2} d \tau+\text { э.c. }\right] \rho_{0}^{\text {las }}
\end{aligned}
$$

где операция эрмитова сопряжения * действует с изменением расположения операторов относительно $\rho_{0}^{\text {las }}$. При переходе от $(19)$ к (21) использована известная формула упорядочивания

$$
e^{-\lambda b^{\dagger} b}=e^{b_{2}^{\dagger} b_{1}\left(e^{-\lambda}-1\right)} .
$$

5 Теоретическая и математическая физика, т. 158, № 3, 2009 г. 
Поскольку множество когерентных векторов всюду плотно в пространстве $l_{2}$, то $\phi_{t}$ однозначно определяет вид супероператора $\varphi_{t}(\cdot) \varphi_{t}^{*}$, действующего на произвольное состояние $\rho_{0}=\rho_{0}^{\text {osc }} \otimes \rho_{0}^{\text {las }}$ :

$$
\phi_{t}(\bar{g}, g, \bar{h}, h) \longrightarrow \varphi_{t} \rho_{0} \varphi_{t}^{*}=\phi_{t}\left(\vec{b}_{2}^{\dagger}, \overleftarrow{b}_{2}, \overleftarrow{b}_{1}^{\dagger}, \vec{b}_{1}\right) \rho_{0}^{\mathrm{osc}}
$$

где индексы и стрелки указывают порядок действия и расположение операторов относительно $\rho_{0}^{\text {osc }}$. Например, стрелка и индекс $\vec{b}{ }_{2}^{\dagger}$ означают, что оператор $b^{\dagger}$ действует на $\rho_{0}^{\text {osc }}$ слева вторым.

В (21) выполним сдвиг $\left\{\vec{z}_{t}, \overleftarrow{z}_{t}^{*}, \vec{w}_{t}, \overleftarrow{w}_{t}^{*}\right\} \rightarrow\left\{\zeta_{t}, \zeta_{t}^{*}, \xi_{t}, \xi_{t}^{*}\right\}$ такой, что

$$
\begin{aligned}
& d \zeta_{t}=-\left(q_{t} \mu_{t}\left(\zeta_{t}-i \vec{\beta}_{t}^{+}\right)+i \sigma_{t}^{2} \vec{\beta}_{t}^{-}\right) d t+\mu_{t} d X_{t}+\sigma_{t} d Y_{t}+f_{\zeta} d t, \\
& d \xi_{t}=-q_{t} \eta_{t}\left(\zeta_{t}-i \vec{\beta}_{t}^{+}\right) d t+\eta_{t} d X_{t}+f_{\xi} d t .
\end{aligned}
$$

Процессы $d \zeta_{t}$ и $d \xi_{t}$ отличаются от $d z_{t}, d w_{t}(18)$ на слагаемое $f d t$ :

$$
\begin{aligned}
f & =\left(\begin{array}{c}
f_{\zeta} \\
f_{\zeta^{*}} \\
f_{\xi} \\
f_{\xi^{*}}
\end{array}\right)=\left(\begin{array}{cc}
\mu_{t} & \sigma_{t} \\
\mu_{t}^{*} & \sigma_{t}^{*} \\
\eta_{t} & 0 \\
\eta_{t}^{*} & 0
\end{array}\right)\left(\begin{array}{c}
q_{t}\left(\zeta_{t}-i \vec{\beta}_{t}^{+}\right)+q_{t}^{*}\left(\zeta_{t}^{*}+i \overleftarrow{\beta}_{t}^{+*}\right) \\
i \sigma_{t} \vec{\beta}_{t}^{-}-i \sigma_{t}^{*} \overleftarrow{\beta}_{t}^{-*}
\end{array}\right)= \\
& =\left(\begin{array}{c}
\mu_{t} q_{t}\left(\zeta_{t}-i \vec{\beta}_{t}^{+}\right)+\mu_{t} q_{t}^{*}\left(\zeta_{t}^{*}+i \overleftarrow{\beta}_{t}^{+*}\right)+i \sigma_{t}^{2} \vec{\beta}_{t}^{-}-i\left|\sigma_{t}\right|^{2} \overleftarrow{\beta}_{t}^{-*} \\
\mu_{t}^{*} q_{t}\left(\zeta_{t}-i \vec{\beta}_{t}^{+}\right)+\mu_{t}^{*} q_{t}^{*}\left(\zeta_{t}^{*}+i \overleftarrow{\beta}_{t}^{+*}\right)+i\left|\sigma_{t}\right|^{2} \vec{\beta}_{t}^{-}-i \sigma_{t}^{* 2} \overleftarrow{\beta}_{t}^{-*} \\
q_{t} \eta_{t}\left(\zeta_{t}-i \vec{\beta}_{t}^{+}\right)+q_{t}^{*} \eta_{t}\left(\zeta_{t}^{*}+i \overleftarrow{\beta}_{t}^{+*}\right) \\
q_{t} \eta_{t}^{*}\left(\zeta_{t}-i \vec{\beta}_{t}^{+}\right)+q_{t}^{*} \eta_{t}^{*}\left(\zeta_{t}^{*}+i \overleftarrow{\beta}_{t}^{+*}\right)
\end{array}\right)
\end{aligned}
$$

Согласно теореме Гирсанова меры $m_{\zeta, \zeta^{*}, \xi, \xi^{*}}$ и $m_{z, z^{*}, w, w^{*}}$ абсолютно непрерывны друг относительно друга, а производная Радона-Никодима $d m_{\zeta, \zeta^{*}, \xi, \xi^{*}} / d m_{z, z^{*}, w, w^{*}}$ имеет вид [13]

$$
\begin{aligned}
& \frac{d m_{\zeta, \zeta^{*}, \xi, \xi^{*}}}{d m_{z, z^{*}, w, w^{*}}}\left(\vec{z}_{t}, \overleftarrow{z}_{t}^{*}, \vec{w}_{t}, \overleftarrow{w}_{t}^{*}\right)=\exp \left[\int_{0}^{t}\left(q_{\tau}\left(\vec{z}_{\tau}-i \vec{\beta}_{\tau}^{+}\right)+\text {э.c. }\right) d X_{\tau}\right] \times \\
& \quad \times \exp \left[\int_{0}^{t}\left(i \sigma_{\tau} \vec{\beta}_{\tau}^{-}+\text {э.c. }\right) d Y_{\tau}\right] \exp \left[-\frac{1}{2} \int_{0}^{t}\left(q_{\tau}\left(\vec{z}_{\tau}-i \vec{\beta}_{\tau}^{+}\right)+\text {э.c. }\right)^{2} d \tau\right] \times \\
& \quad \times \exp \left[-\frac{1}{2} \int_{0}^{t}\left(i \sigma_{\tau} \vec{\beta}_{\tau}^{-}+\text {э.c. }\right)^{2} d \tau\right]
\end{aligned}
$$

Следовательно, имеет место равенство $M \phi_{t}=M \psi_{t}$, где

$$
\begin{aligned}
\psi_{t} \stackrel{\text { def }}{=} & \exp \left[\int_{0}^{t}\left|q_{\tau}\left(\zeta_{\tau}-i \vec{\beta}+t\right)\right|^{2} d \tau\right] \exp \left[\int_{0}^{t}\left|\sigma_{\tau} \vec{\beta}_{\tau}^{-}\right|^{2} d \tau\right] \times \\
& \times \exp \left[\bar{g} \zeta_{t}-\frac{\bar{g}^{2}}{2} \int_{0}^{t}\left(\mu_{\tau}^{2}+\sigma_{\tau}^{2}\right) d \tau+\text { э.c. }\right] \times \\
& \times \exp \left[\bar{g} h\left(e^{-\int_{0}^{t} q_{\tau} \mu_{\tau} d \tau}-1\right)+\text { э.c. }\right] \exp \left[h \xi_{t}-\frac{h^{2}}{2} \int_{0}^{t} \eta_{\tau}^{2} d \tau+\text { э.c. }\right] \rho_{0}^{\text {las }},
\end{aligned}
$$


а процессы $\zeta_{t}, \xi_{t}$ удовлетворяют системе уравнений

$$
\begin{array}{ll}
d \zeta_{t}=\mu_{t} q_{t}^{*}\left(\zeta_{t}^{*}+i \overleftarrow{\beta}_{t}^{+*}\right) d t-i\left|\sigma_{t}\right|^{2 \overleftarrow{\beta}}{ }_{t}^{*} d t+\mu_{t} d X_{t}+\sigma_{t} d Y_{t}, & \zeta_{0}=0 \\
d \xi_{t}=q_{t}^{*} \eta_{t}\left(\zeta_{t}^{*}+i \overleftarrow{\beta}_{t}^{+*}\right) d t+\eta_{t} d X_{t}, & \xi_{0}=0
\end{array}
$$

Соответственно $\zeta_{t}^{*}$ и $\xi_{t}^{*}$ эрмитово сопряжены $\zeta_{t}$ и $\xi_{t}$ с учетом изменения расположения операторов относительно $\rho_{0}^{\text {las }}$.

Рассмотрим математическое ожидание $\mathrm{M} \psi_{t}(23)$. Введем для этого случайную функцию времени

$$
v=v_{t}^{\alpha, \beta}=\exp \left[\int_{0}^{t}\left|q_{\tau}\right|^{2}\left(i \overleftarrow{\beta}_{\tau}^{+*} \zeta_{\tau}-i \vec{\beta}_{\tau}^{+} \zeta_{\tau}^{*}+\zeta_{\tau} \zeta_{\tau}^{*}\right) d \tau\right] e^{\alpha \bar{g} \zeta_{t}} e^{\beta g \zeta_{t}^{*}} e^{h \xi_{t}} e^{\bar{h} \xi_{t}^{*}}
$$

зависящую от параметров $\alpha$ и $\beta$. При этом $\mathrm{M} \psi_{t}$ принимает вид

$$
\begin{aligned}
\mathrm{M} \psi_{t}= & \exp \left[\int_{0}^{t}\left(\left|q_{\tau} \vec{\beta}+\right|^{2}+\left|\sigma_{\tau} \vec{\beta}_{\tau}^{-}\right|^{2}\right) d \tau\right] \exp \left[-\frac{\bar{g}^{2}}{2} \int_{0}^{t}\left(\mu_{\tau}^{2}+\sigma_{\tau}^{2}\right) d \tau+\text { э.c. }\right] \times \\
& \times \exp \left[\bar{g} h\left(\exp \left[-\int_{0}^{t} q_{\tau} \mu_{\tau} d \tau\right]-1\right)+\text { э.c. }\right] \times \\
& \times \exp \left[-\frac{h^{2}}{2} \int_{0}^{t} \eta_{\tau}^{2} d \tau+\text { э.c. }\right] \mathrm{M}\left\{\left.v_{t}^{\alpha, \beta}\right|_{\substack{\alpha=1 \\
\beta=1}}\right\} \rho_{0}^{\text {las }} .
\end{aligned}
$$

Рассмотрим вывод дифференциального уравнения для $\operatorname{M}\left\{v_{t}^{\alpha, \beta}\right\}$. Заметим, что

$$
\mathrm{M}\left\{\zeta_{t} v\right\}=\frac{1}{\bar{g}} \frac{\partial \mathrm{M}\{v\}}{\partial \alpha}, \quad \mathrm{M}\left\{\zeta_{t}^{*} v\right\}=\frac{1}{g} \frac{\partial \mathrm{M}\{v\}}{\partial \beta}, \quad \mathrm{M}\left\{\zeta_{t} \zeta_{t}^{*} v\right\}=\frac{1}{|g|^{2}} \frac{\partial^{2} \mathrm{M}\{v\}}{\partial \alpha \partial \beta}
$$

и в соответствии с определениями процессов $\zeta_{t}, \xi_{t}(24)$ имеем

$$
\begin{aligned}
\frac{\partial \mathrm{M}\{v\}}{\partial t}= & \frac{\left|q_{t}\right|^{2}}{|g|^{2}} \frac{\partial^{2} \mathrm{M}\{v\}}{\partial \alpha \partial \beta}+\frac{i\left|q_{t}\right|^{2} \overleftarrow{\beta}_{t}^{+*}}{\bar{g}} \frac{\partial \mathrm{M}\{v\}}{\partial \alpha}-\frac{i\left|q_{t}\right|^{2} \vec{\beta}_{t}^{+}}{g} \frac{\partial \mathrm{M}\{v\}}{\partial \beta}+ \\
& +\alpha \bar{g}\left(\left(i \mu_{t} q_{t}^{*} \overleftarrow{\beta}_{t}^{+*}-i\left|\sigma_{t}\right|^{2} \overleftarrow{\beta}_{t}^{-*}\right) \mathrm{M}\{v\}+\frac{\mu_{t} q_{t}^{*}}{g} \frac{\partial \mathrm{M}\{v\}}{\partial \beta}\right)+ \\
& +\frac{\alpha^{2}}{2} \bar{g}^{2}\left(\mu_{t}^{2}+\sigma_{t}^{2}\right) \mathrm{M}\{v\}+h q_{t}^{*} \eta_{t}\left(i \overleftarrow{\beta}_{t}^{+*} \mathrm{M}\{v\}+\frac{1}{g} \frac{\partial \mathrm{M}\{v\}}{\partial \beta}\right)+ \\
& +\beta g\left(\left(-i \mu_{t}^{*} q_{t} \vec{\beta}_{t}^{+}+i\left|\sigma_{t}\right|^{2} \vec{\beta}_{t}^{-}\right) \mathrm{M}\{v\}+\frac{\mu_{t}^{*} q_{t}}{\bar{g}} \frac{\partial \mathrm{M}\{v\}}{\partial \alpha}\right)+ \\
& +\frac{\beta^{2}}{2} g^{2}\left(\mu_{t}^{* 2}+\sigma_{t}^{* 2}\right) \mathrm{M}\{v\}+\bar{h} q_{t} \eta_{t}^{*}\left(-i \vec{\beta}+\mathrm{M}\{v\}+\frac{1}{\bar{g}} \frac{\partial \mathrm{M}\{v\}}{\partial \alpha}\right)+ \\
& +\alpha \beta|g|^{2}\left(\left|\mu_{t}\right|^{2}+\left|\sigma_{t}\right|^{2}\right) \mathrm{M}\{v\}+h^{2} \frac{\eta_{t}^{2}}{2} \mathrm{M}\{v\}+\bar{h}^{2} \frac{\eta_{t}^{* 2}}{2} \mathrm{M}\{v\}+ \\
& +|h|^{2}\left|\eta_{t}\right|^{2} \mathrm{M}\{v\}+\alpha \bar{g} h \mu_{t} \eta_{t} \mathrm{M}\{v\}+\alpha \bar{g} \bar{h} \mu_{t} \eta_{t}^{*} \mathrm{M}\{v\}+ \\
& +\beta h g \mu_{t}^{*} \eta_{t} \mathrm{M}\{v\}+\beta \bar{h} g \mu_{t}^{*} \eta_{t}^{*} \mathrm{M}\{v\}
\end{aligned}
$$

Поскольку при вычислении $\mathrm{M} \psi_{t}(25)$ параметры $\alpha$ и $\beta$ полагаются равными единице, нас будет интересовать частное решение этого уравнения, которое имеет вид экспоненты от квадратичной функции переменных $g$ и $h$. После нескольких подстановок 
с учетом $(13),(14)$ и (25) для математического ожидания М $\psi_{t}$ получаем

$$
\begin{aligned}
& \mathrm{M} \psi_{t}=\exp \left[\int_{0}^{t}\left(\left|q_{\tau} \vec{\beta}_{\tau}^{+}\right|^{2}+\left|\sigma_{\tau} \vec{\beta}_{\tau}^{-}\right|^{2}+\left|q_{\tau} f_{2}(\tau)\right|^{2}+\left|q_{\tau}\right|^{2} f_{1}(\tau)\right) d \tau\right] \times \\
& \times \exp \left[\int_{0}^{t}\left(i\left|q_{\tau}\right|^{2} \overleftarrow{\beta}_{\tau}^{+*} f_{2}(\tau)+\text { э.c. }\right) d \tau\right] \times \\
& \times e^{|g|^{2} f_{1}(t)} e^{\bar{g} f_{2}(t)+\text { э.c. }} \exp \left[(\bar{g} h+\text { э.c. })\left(e^{\int_{0}^{t}\left|q_{\tau}\right|^{2} f_{1}(\tau) d \tau}-1\right)\right] \times \\
& \times \exp \left[h \int_{0}^{t}\left|q_{\tau}\right|^{2} e^{\int_{0}^{\tau}\left|q_{s}\right|^{2} f_{1}(s) d s}\left(f_{2}^{*}(\tau)+i \overleftarrow{\beta}_{\tau}^{+*}\right) d \tau+\text { э.c. }\right] \times \\
& \times \exp \left[|h|^{2} \int_{0}^{t}\left|q_{\tau}\right|^{2} e^{2 \int_{0}^{\tau}\left|q_{s}\right|^{2} f_{1}(s) d s} d \tau\right] \rho_{0}^{\text {las }},
\end{aligned}
$$

где

$$
\begin{aligned}
& f_{1}(t)=\frac{\nu\left(1-e^{-\lambda t}\right)}{\nu+1-\nu e^{-\lambda t}} e^{\lambda(2 \nu+1) t} \\
& f_{2}(t)=-i \int_{0}^{t} \exp \left[\int_{\tau}^{t}\left|q_{s}\right|^{2} f_{1}(s) d s\right]\left(\left|q_{\tau}\right|^{2} f_{1}(\tau) \vec{\beta}_{\tau}^{+}+\left|\sigma_{\tau}\right|^{2} \overleftarrow{\beta}_{\tau}^{-*}\right) d \tau
\end{aligned}
$$

причем с учетом (9) $\left|q_{t}\right|^{2}=\lambda(\nu+1) e^{-\lambda(2 \nu+1) t}$ и $\left|\sigma_{t}\right|^{2}=\lambda \nu e^{\lambda(2 \nu+1) t}$.

По определению $(22)$ вид супероператора $\mathrm{M} \varphi_{t}(\cdot) \varphi_{t}^{*}$, действующего на произвольное состояние $\rho_{0}$, восстанавливается из $\mathrm{M} \psi_{t}(26)$ с помощью подстановки

$$
\{\bar{g}, g, \bar{h}, h\} \rightarrow\left\{\vec{b}_{2}^{\dagger}, \overleftarrow{b}_{2}, \overleftarrow{b}_{1}^{\dagger}, \vec{b}_{1}\right\}
$$

В силу (19) и (20) после коммутаций и подстановки функций $\beta_{t}^{ \pm}, C_{t}(10), f_{1}(t)(27)$ и $f_{2}(t)(28)$ получаем упорядоченное решение уравнения (5)

$$
\begin{aligned}
\rho_{t}= & \mathrm{M} u_{t} \rho_{0} u_{t}^{*}=e^{-\lambda \nu t} e^{-i \omega a^{\dagger} a t} e^{-C_{t}} \exp \left[-b^{\dagger} b\left(\frac{\lambda}{2}(2 \nu+1)+i \Omega\right) t\right] e^{-i b^{\dagger} \beta_{t}^{+}} e^{-i \beta_{t}^{-} b} \times \\
& \times \mathrm{M} \psi_{t}\left(\vec{b}_{2}^{\dagger}, \vec{b}_{1}, \overleftarrow{b}_{1}^{\dagger}, \overleftarrow{b}_{2}\right) \rho_{0}^{\text {osc }} e^{i \beta_{t}^{-*} b^{\dagger}} e^{i \beta_{t}^{+*} b} \times \\
& \times \exp \left[-b^{\dagger} b\left(\frac{\lambda}{2}(2 \nu+1)-i \Omega\right) t\right] e^{-C_{t}^{*}} e^{i \omega a^{\dagger} a t}= \\
= & \frac{1}{\alpha_{t}} e^{-i \omega\left(\overrightarrow{a^{\dagger} a}-\overleftrightarrow{a^{\dagger} a}\right) t} e^{-\left|\beta_{t}\right|^{2} / \alpha_{t}} \exp \left[g\left(\overleftarrow{a^{\dagger} a}-{\overrightarrow{a^{\dagger} a}}^{\dagger}\right) \int_{0}^{t}\left(\beta_{\tau}-\beta_{\tau}^{*}\right) d \tau\right] e^{\vec{b}_{3}^{\dagger} \overleftarrow{b}_{3}\left(1-1 / \alpha_{t}\right)} \times \\
& \times \exp \left[-i \vec{b}_{3}^{\dagger} \frac{\beta_{t}}{\alpha_{t}}+i \overleftarrow{b}_{3} \frac{\beta_{t}^{*}}{\alpha_{t}}\right] \times \\
& \times \exp \left[-\left(\vec{b}^{\dagger} b_{2}+\overleftrightarrow{\left.b^{\dagger} b_{2}\right)}\left(\frac{\lambda t}{2}+\ln \alpha_{t}\right)-i \Omega\left(\overrightarrow{b^{\dagger} b_{2}}-\overleftarrow{b^{\dagger} b_{2}}\right) t\right] \times\right. \\
& \times \exp \left[\vec{b}_{1} \overleftarrow{b}_{1}^{\dagger}\left(1-\frac{e^{-\lambda t}}{\alpha_{t}}\right)\right] \times \\
& \times \exp \left[-i \vec{b}_{1}\left(\frac{\beta_{t}^{*}}{\alpha_{t}} e^{-(i \Omega+\lambda / 2) t}+g\left(\overrightarrow{a^{\dagger} a}-\overleftrightarrow{a^{\dagger} a}\right) \frac{e^{-(i \Omega+\lambda / 2) t}-1}{-i \Omega-\lambda / 2}\right)\right] \times \\
& \times \exp \left[i \overleftarrow{b}_{1}^{\dagger}\left(\frac{\beta_{t}}{\alpha_{t}} e^{(i \Omega-\lambda / 2) t}-g\left(\overrightarrow{a^{\dagger} a}-\overleftrightarrow{a^{\dagger} a}\right) \frac{e^{(i \Omega-\lambda / 2) t}-1}{i \Omega-\lambda / 2}\right)\right] \rho_{0},
\end{aligned}
$$


где

$$
\begin{aligned}
& \alpha_{t}=\nu+1-\nu e^{-\lambda t} \\
& \beta_{t}=e^{-(i \Omega+\lambda / 2) t} \int_{0}^{t}\left(\overleftarrow{g a^{\dagger} a}+f_{\tau}+g\left(\overline{a^{\dagger} a}-\overleftarrow{a^{\dagger} a}\right) \alpha_{\tau}\right) e^{(i \Omega+\lambda / 2) \tau} d \tau .
\end{aligned}
$$

Таким образом, формула (29) задает явный вид полугруппы $T_{t}(\cdot): \rho_{t}=\mathcal{T}_{t}\left(\rho_{0}\right)$ для уравнения (5).

\section{4. РЕДУЦИРОВАННЫЕ МАТРИЦЫ ПЛОТНОСТИ ОСЦИЛЛЯТОРА И ЛАЗЕРНОГО ИЗЛУЧЕНИЯ}

Рассмотрим решение (29), полученное в разделе 3 . В наиболее простом случае свободного осциллятора, $H=\hbar \Omega b^{\dagger} b$, матрица плотности $\rho_{t}$ принимает вид

$$
\begin{aligned}
\rho_{t}= & \frac{1}{\alpha_{t}} \exp \left[\vec{b}_{3}^{\dagger} \overleftarrow{b}_{3}\left(1-\frac{1}{\alpha_{t}}\right)\right] \exp \left[-\left({\overrightarrow{b^{\dagger} b_{2}}}+{\overleftarrow{b^{\dagger}}}_{2}\right)\left(\frac{\lambda t}{2}+\ln \alpha_{t}\right)-i \Omega\left({\overrightarrow{b^{\dagger}}}_{2}-\overleftarrow{b^{\dagger} b_{2}}\right) t\right] \times \\
& \times \exp \left[\vec{b}_{1} \overleftarrow{b_{1}^{\dagger}}\left(1-\frac{e^{-\lambda t}}{\alpha_{t}}\right)\right] \rho_{0} .
\end{aligned}
$$

Нетрудно видеть, что

$$
\frac{\lambda t}{2}+\ln \alpha_{t}=\frac{\lambda t}{2}+\ln \left\{1+\nu-\nu e^{-\lambda t}\right\} \rightarrow \infty \quad \text { при } \quad t \rightarrow \infty .
$$

Следовательно, множитель $\exp \left[-\left({\overrightarrow{b^{\dagger}}}_{2}+{\overleftarrow{b^{\dagger}}}_{2}\right)\left(\lambda t / 2+\ln \alpha_{t}\right)\right]$ переводит любое состояние с течением времени в вакуумное, и состояние осциллятора становится стационарным гиббсовским:

$$
\begin{aligned}
\rho_{\infty} & =\frac{1}{\alpha_{t}} e^{\overrightarrow{b^{\dagger}} \overleftarrow{b}\left(1-1 / \alpha_{t}\right)}|0\rangle\left\langle 0\left|=\frac{1}{\alpha_{t}} \sum_{n=0}^{\infty} \frac{\left(1-1 / \alpha_{t}\right)^{n}}{n !}\left(b^{\dagger}\right)^{n}\right| 0\right\rangle\langle 0| b^{n}= \\
& =\frac{1}{\nu+1} \sum_{n=0}^{\infty}\left(\frac{\nu}{\nu+1}\right)^{n}|n\rangle\langle n| \quad \text { при } \quad \lambda t \gg 1 .
\end{aligned}
$$

При этом, как отмечалось в разделе 1 , параметр $\lambda$ характеризует величину связи осциллятора с термостатом.

Поскольку в состоянии равновесия температура осциллятора и окружения равны, параметр $\nu$ характеризует температуру окружения $T$ :

$$
\frac{\nu}{\nu+1}=e^{-\hbar \Omega /(k T)}, \quad \nu=\frac{1}{e^{\hbar \Omega /(k T)}-1} .
$$

Точнее говоря, $\nu$ с точностью до размерного множителя имеет смысл средней энергии свободного осциллятора в состоянии равновесия с окружением

$$
\left\langle b^{\dagger} b\right\rangle=\operatorname{Tr}\left(b^{\dagger} b \rho_{\infty}\right)=\nu
$$


Вернемся к исходной модели. Состояние осциллятора есть частичный след оператора $\rho_{t}(29)$ по состояниям лазерного излучения:

$$
\begin{aligned}
\rho_{t}^{\text {osc }}= & \operatorname{Tr}_{\text {las }}\left(\rho_{t}\right)=\frac{1}{\alpha_{t}} \sum_{n=0}^{\infty} e^{-\left|\beta_{n}(t)\right|^{2} / \alpha_{t}} e^{\vec{b}_{3}^{\dagger} \overleftarrow{b}_{3}\left(1-1 / \alpha_{t}\right)} \exp \left[-i \vec{b}_{3}^{\dagger} \frac{\beta_{n}(t)}{\alpha_{t}}+i \overleftarrow{b}_{3} \frac{\beta_{n}^{*}(t)}{\alpha_{t}}\right] \times \\
& \times \exp \left[-\left({\overrightarrow{b^{\dagger}}}_{2}+\overleftarrow{b^{\dagger} b_{2}}\right)\left(\frac{\lambda t}{2}+\ln \alpha_{t}\right)-i \Omega\left(\vec{b}^{\dagger} b_{2}-\overleftarrow{b^{\dagger}} b_{2}\right) t\right] e^{\vec{b}_{1} \overleftarrow{b}_{1}^{\dagger}\left(1-e^{-\lambda t} / \alpha_{t}\right)} \times \\
& \times \exp \left[-i \vec{b}_{1} \frac{\beta_{n}^{*}(t)}{\alpha_{t}} e^{-(i \Omega+\lambda / 2) t}+i{\overleftarrow{b^{\dagger}}}_{1} \frac{\beta_{n}(t)}{\alpha_{t}} e^{(i \Omega-\lambda / 2) t}\right] \rho_{0}^{\text {osc }} \rho_{n n}^{\text {las }}(0)
\end{aligned}
$$

где

$$
\beta_{n}(t)=e^{-(i \Omega+\lambda / 2) t} \int_{0}^{t}\left(g n+f_{\tau}\right) e^{(i \Omega+\lambda / 2) \tau} d \tau
$$

а начальное состояние системы "осциллятор-электромагнитное поле" имеет вид тензорного произведения: $\rho_{0}=\rho_{0}^{\text {osc }} \otimes \rho_{0}^{\text {las }}$ с диагональными элементами матрицы плотности электромагнитного поля $\rho_{n n}^{\text {las }}(0)$. Выполним преобразование формулы $(33)$. Изменим порядок следования операторов

$$
\exp \left[-i \vec{b}_{1} \frac{\beta_{n}^{*}(t)}{\alpha_{t}} e^{-(i \Omega+\lambda / 2) t}+i \overleftarrow{b}^{\dagger}{ }_{1} \frac{\beta_{n}(t)}{\alpha_{t}} e^{(i \Omega-\lambda / 2) t}\right]
$$

и

$$
e^{{\overrightarrow{b^{\dagger}}}_{3} \overleftarrow{b}_{3}\left(1-1 / \alpha_{t}\right)} \exp \left[-\left({\overrightarrow{b^{\dagger}}}_{2}+\overleftarrow{b^{\dagger} b_{2}}\right)\left(\frac{\lambda t}{2}+\ln \alpha_{t}\right)-i \Omega\left({\overrightarrow{b^{\dagger} b_{2}}}_{-} \overleftarrow{b^{\dagger} b_{2}}\right) t\right]
$$

Полагая

$$
U_{n}=e^{-i b \beta_{n}^{*}(t)-i b^{\dagger} \beta_{n}(t)},
$$

имеем

$$
\begin{aligned}
\rho_{t}^{\text {osc }}= & \sum_{n=0}^{\infty} U_{n} \frac{1}{\alpha_{t}} e^{{\overrightarrow{b^{\dagger}}}_{3} \overleftarrow{b}_{3}\left(1-1 / \alpha_{t}\right)} \exp \left[-\left({\overrightarrow{b^{\dagger}}}_{2}+\overleftarrow{b^{\dagger}} b_{2}\right)\left(\frac{\lambda t}{2}+\ln \alpha_{t}\right)\right] \times \\
& \times e^{-i \Omega\left({\overrightarrow{b^{\dagger} b_{2}}}-\overleftarrow{b^{\dagger} b_{2}}\right) t} e^{\vec{b}_{1} \overleftarrow{b_{1}^{\dagger}}\left(1-e^{-\lambda t} / \alpha_{t}\right)} \rho_{0}^{\text {osc }} U_{n}^{+} \rho_{n n}^{\text {las }}(0) .
\end{aligned}
$$

При больших временах $\lambda t \gg 1$ состояние осциллятора представляет собой унитарное преобразование (вращение) стационарного гиббсовского состояния

$$
\rho_{\infty}^{\mathrm{osc}}=\sum_{n, m=0}^{\infty} U_{n} \frac{1}{\nu+1}\left(\frac{\nu}{\nu+1}\right)^{m}|m\rangle_{\mathrm{osc} \text { osc }}\langle m| U_{n}^{\dagger} \rho_{n n}^{\mathrm{las}}(0)
$$

Заметим, что $U_{n}$ зависит от внешней силы $f_{t}$ и давления электромагнитного поля на осциллятор, но не зависит от температуры окружения.

Лазерный луч, отражаясь от подвижного зеркала, несет информацию о его состоянии. В экспериментах измеряется фаза излучения на выходе из полости. Для теоретического расчета результатов измерений необходимо вычислить редуцированную матрицу плотности лазерного излучения. Рассчитаем ее для начального состояния 
$\rho_{0}=|0\rangle\left\langle\left. 0\right|_{\text {osc }} \otimes \rho_{\text {las }}(0)\right.$, не конкретизируя начальное состояние лазерного излучения на входе в полость. При этом

$$
\begin{aligned}
\rho_{t}= & \frac{1}{\alpha_{t}} \exp \left[-i \overrightarrow{b^{\dagger}} \frac{\beta_{t}}{\alpha_{t}}+i \overleftarrow{b} \frac{\beta_{t}^{*}}{\alpha_{t}}\right] e^{\overrightarrow{b^{\dagger}} \overleftarrow{b}\left(1-1 / \alpha_{t}\right)} e^{-i \omega\left(\overrightarrow{a^{\dagger} a}-\overleftarrow{a^{\dagger} a}\right) t} e^{-\left|\beta_{t}\right|^{2} / \alpha_{t}} \times \\
& \times \exp \left[g\left(\overleftarrow{a^{\dagger} a}-\overrightarrow{a^{\dagger} a}\right) \int_{0}^{t}\left(\beta_{\tau}-\beta_{\tau}^{*}\right) d \tau\right]|0\rangle\left\langle\left. 0\right|_{\text {osc }} \otimes \rho(0)_{\text {las }} .\right.
\end{aligned}
$$

Частичный след $\rho_{t}$ по состояниям осциллятора $\rho_{\text {las }}=\operatorname{Tr}_{\text {osc }}\left(\rho_{t}\right)$ можно вычислить с помощью производящей функции полиномов Лагерра [14]:

$$
\begin{aligned}
& \operatorname{Tr}\left(e^{z \overrightarrow{b^{\dagger}}+z^{*} \overleftarrow{b}} e^{c \overrightarrow{b^{\dagger}} \overleftarrow{b}}|0\rangle\langle 0|\right)=\sum_{n, m=0}^{\infty}\left\langle m\left|e^{z b^{\dagger}}\right| n\right\rangle c^{n}\left\langle n\left|e^{z^{*} b}\right| m\right\rangle= \\
& \quad=\sum_{n, m=0}^{\infty} \sum_{k, k^{\prime}=0}^{m}\left\langle m-k^{\prime}\right| \frac{z^{k^{\prime}}}{k^{\prime} !} \sqrt{\frac{m !}{\left(m-k^{\prime}\right) !}|n\rangle c^{n}\left\langle n\left|\frac{\left(z^{*}\right)^{k}}{k !} \sqrt{\frac{m !}{(m-k) !}}\right| m-k\right\rangle=} \\
& =\sum_{m=0}^{\infty} \sum_{k=0}^{m} \frac{|z|^{2 k}}{c^{k}} c^{m} \frac{m !}{(m-k) ! k ! k !}=\sum_{m=0}^{\infty} L_{m}\left(-\frac{|z|^{2}}{c}\right) c^{m}=\frac{e^{|z|^{2} /(1-c)}}{1-c} .
\end{aligned}
$$

Таким образом, мы имеем

$$
\rho(t)_{\mathrm{las}}=e^{-i \omega\left(\overrightarrow{a^{\dagger} a}-\overleftrightarrow{a^{\dagger} a}\right) t} \exp \left[g\left(\overleftarrow{a^{\dagger} a}-\overrightarrow{a^{\dagger} a}\right) \int_{0}^{t}\left(\beta_{\tau}-\beta_{\tau}^{*}\right) d \tau\right] \rho(0)_{\mathrm{las}}
$$

где

$$
\beta_{t}=e^{-(i \Omega+\lambda / 2) t} \int_{0}^{t}\left(g \overleftarrow{a^{\dagger} a}+f_{\tau}+g\left(\overrightarrow{a^{\dagger} a}-\overleftarrow{a^{\dagger} a}\right) \alpha_{\tau}\right) e^{(i \Omega+\lambda / 2) \tau} d \tau
$$

Информация о состоянии осциллятора записывается во внедиагональные элементы матрицы плотности $\rho(t)_{\text {las. }}$ При определенном способе измерения фазы волны, например с помощью интерференционного метода, можно рассчитать сигнал на выходе из полости.

\section{5. ИНТЕРФЕРЕНЦИОННЫЙ МЕТОД ИЗМЕРЕНИЯ ФАЗЫ}

Рассмотрим интерференцию электромагнитного излучения, выходящего из двух полостей с подвижными отражающими стенками (см. рисунок). Лазерное излучение попадает на сплиттер (BS1), делящий пучок в отношении $50 / 50$, и распадается на два луча. В отраженный и прошедший лучи помещены полости с подвижными стенками. На втором сплиттере (BS2), который также делит пучок в отношении 50/50, лучи складываются. Предварительно фаза волны в первом луче сдвигается на 90. За вторым сплиттером на выходе интерферометра (PD1, PD2 - фотодетекторы) измеряется разность интенсивностей двух лучей.

Унитарные матрицы рассеяния первого и второго сплиттеров имеют вид [15] $S_{1,2}=(1 / \sqrt{2})\left(\begin{array}{cc}1 & i \\ i & 1\end{array}\right)$. Если на входе интерферометра излучение находится в когерентном состоянии

$$
|\psi\rangle=|z\rangle=e^{-|z|^{2} / 2}\left(1, z, \frac{z^{2}}{\sqrt{2 !}}, \ldots, \frac{z^{n}}{\sqrt{n !}}, \ldots\right)
$$




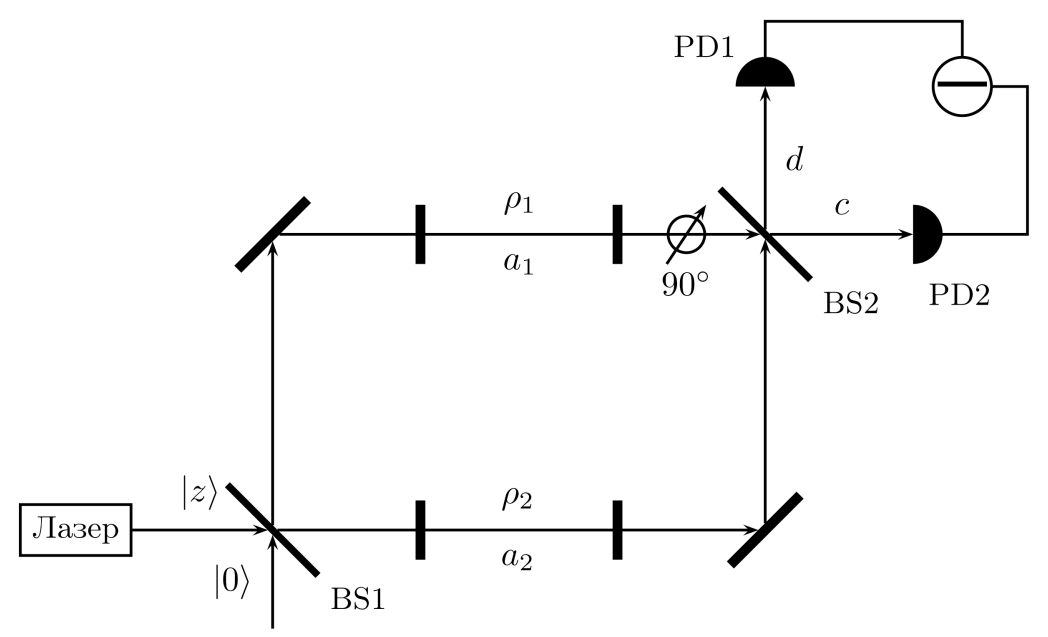

Ход лучей и эволюции состояния лазерного излучения в двухплечевом интерферометре.

с амплитудой $z \in \mathbb{C}$ и средним числом фотонов $N=|z|^{2}$, то после первого сплиттера на входе в полости отраженный и прошедший лучи будут находится в состояниях

$$
\rho(0)_{1}=\left|\frac{i z}{\sqrt{2}}\right\rangle\left\langle-\frac{i z}{\sqrt{2}}\left|, \quad \rho(0)_{2}=\right| \frac{z}{\sqrt{2}}\right\rangle\left\langle\frac{z}{\sqrt{2}}\right| .
$$

Состояние излучения в полостях представляется оператором (36). Для дальнейших вычислений удобно записать матрицу плотности системы в каноническом базисе:

$$
\begin{aligned}
\rho_{1}(t)=e^{-N / 2} \sum_{n=0}^{\infty} \sum_{m=0}^{\infty}|n\rangle_{11}\langle m| \frac{(i z)^{n}\left(-i z^{*}\right)^{m}}{2^{n / 2} 2^{m / 2} \sqrt{n ! m !}} e^{-i \omega(n-m) t} \times \\
\times \exp \left[g^{2}(m-n) \int_{0}^{t} \int_{0}^{\tau} d \tau d s\left(m e^{(i \Omega+\lambda / 2)(s-\tau)}-n e^{(-i \Omega+\lambda / 2)(s-\tau)}\right)\right] \times \\
\times \exp \left[-2 g^{2}(m-n)^{2} \int_{0}^{t} \int_{0}^{\tau} d \tau d s \alpha_{s} e^{\lambda(s-\tau) / 2} \cos \Omega(s-\tau)\right] \times \\
\quad \times \exp \left[2 i g(m-n) \int_{0}^{t} \int_{0}^{\tau} d \tau d s f_{s} e^{\lambda(s-\tau) / 2} \sin \Omega(s-\tau)\right]
\end{aligned}
$$

Будем считать, что сила $F(t)$ действует на стенку второй полости в противофазе с такой же силой $F(t)$, действующей на стенку первой полости. Тогда с точностью до преобразования $z \rightarrow i z$ (см. (37)) состояние $\rho_{2}(t)$ излучения во второй полости определяется формулой (38) со знаком минус перед $f_{t}$.

Наблюдаемая, описывающая сигнал на выходе, с точностью до размерного числового множителя равна

$$
\hat{I}=c^{\dagger} c-d^{\dagger} d
$$


где операторы $c$ и $d$ соответствуют выходящим лучам и выражаются, с учетом дополнительного сдвига фазы на 90 , через операторы, описывающие излучение внутри интерферометра:

$$
c=\frac{i}{\sqrt{2}}\left(a_{1}+a_{2}\right), \quad d=\frac{1}{\sqrt{2}}\left(-a_{1}+a_{2}\right),
$$

где операторы $a_{1}$ и $a_{2}$ соответствуют излучению в первой и второй полостях. Следовательно, наблюдаемая выходного сигнала, выраженная через операторы, действующие в пространстве состояний лучей внутри интерферометра, равна

$$
\hat{I}=a_{1}^{\dagger} \otimes a_{2}+a_{1} \otimes a_{2}^{\dagger}
$$

Среднее значение наблюдаемой (39) и ее дисперсия имеют вид

$$
\begin{gathered}
I(t)=\operatorname{Tr}\left(\hat{I} \rho_{t}\right), \quad D(t)=\operatorname{Tr}\left(\hat{I}^{2} \rho_{t}\right)-I^{2}(t), \\
\hat{I}^{2}=\left(a_{1}^{\dagger}\right)^{2} \otimes\left(a_{2}\right)^{2}+2 a_{1}^{\dagger} a_{1} \otimes a_{2}^{\dagger} a_{2}+a_{2}^{\dagger} a_{2}+a_{1}^{\dagger} a_{1}+\left(a_{1}\right)^{2} \otimes\left(a_{2}^{\dagger}\right)^{2},
\end{gathered}
$$

где $\rho_{t}=\rho_{1}(t) \otimes \rho_{2}(t)(38)$ - состояние излучения внутри интерферометра, и вычисляются явно:

$$
\begin{aligned}
I(t) & =N \exp \left[-2 g^{2} c_{t}^{\nu}-2 N \sin ^{2}\left(g^{2} s_{t}\right)\right] \sin \left(4 g \theta_{t}\right) \approx 4 N g \theta_{t}, \\
D(t) & =N+\frac{N^{2}}{2}-\frac{N^{2}}{2} \exp \left[-8 g^{2} c_{t}^{\nu}-2 N \sin ^{2}\left(2 g^{2} s_{t}\right)\right] \cos \left(8 g \theta_{t}\right)-I(t)^{2} \approx \\
& \approx N+4 N^{2} g^{2} c_{t}^{\nu}+4 N^{3} g^{4} s_{t}^{2},
\end{aligned}
$$

где

$$
\begin{aligned}
\theta_{t} & =\int_{0}^{t} \int_{0}^{\tau} d \tau d s f_{s} e^{\lambda(s-\tau) / 2} \sin \Omega(s-\tau), \\
c_{t}^{\nu} & =\int_{0}^{t} \int_{0}^{\tau} d \tau d s\left(2 \alpha_{s}-1\right) e^{\lambda(s-\tau) / 2} \cos \Omega(s-\tau), \\
s_{t} & =\int_{0}^{t} \int_{0}^{\tau} d \tau d s e^{\lambda(s-\tau) / 2} \sin \Omega(s-\tau) .
\end{aligned}
$$

Приближенные выражения для (40), (41) справедливы при учете малости силы $g \theta_{t} \ll 1$ и малости константы взаимодействия лазерного луча и осциллятора $g^{2} c_{t}^{\nu} \ll 1$, $g^{2} s_{t} \ll 1$. Характерно, что в этом приближении среднее значение сигнала определяется только воздействием силы $f_{t}$ на осциллятор (см. (40), (42)). Однако, как следует из (44), функция $\left|s_{t}\right|$ является возрастающей функцией времени, поэтому в области $N g^{4} s_{t}^{2} \gg 1$ разложения (40), (41) неприменимы, и $I(t) \rightarrow 0, D(t) \rightarrow N^{2} / 2$ экспоненциально по $\mathrm{Ng}^{4} s_{t}^{2}$.

Рассмотрим относительную дисперсию $\sigma_{t}^{2}=D(t) / I^{2}(t)$; если $\sigma_{t}^{2} \leqslant 1$, то сигнал может быть обнаружен на фоне шума. Из формул $(40),(41)$ следует, что квадрат относительных флуктуаций состоит из трех слагаемых

$$
\sigma^{2}(t)=\frac{D(t)}{I^{2}(t)} \approx \frac{1}{16 g^{2} \theta_{t}^{2} N}+\frac{c_{t}^{\nu}}{4 \theta_{t}^{2}}+\frac{g^{2} s_{t}^{2} N}{4 \theta_{t}^{2}} \leqslant 1 .
$$


Анализ первого и третьего слагаемых приведен в работе [16]. Температура окружения влияет только на второй член в $\sigma^{2}$, не зависящий от числа фотонов в полости и ее параметров, т.е. на стандартный квантовый предел [17].

В разложении по малому параметру $\lambda$ функции $\alpha_{t}$, которая входит в $c_{t}^{\nu}(\mathrm{cm} .(4))$, с учетом (32) имеем

$$
\alpha_{t}=\nu+1-\nu e^{-\lambda t} \approx 1+\lambda \nu t=1+\frac{k T \lambda t}{\hbar \Omega} \quad \text { при } \quad \lambda t \ll 1
$$

для собственной частоты колебаний макроскопического маятника $\Omega /(2 \pi) \sim O(1) \mathrm{c}^{-1}$. Следовательно, температура окружения не будет влиять на точность измерений, если выполнено условие

$$
\frac{k T \lambda t}{\hbar \Omega} \ll 1,
$$

которое для температуры $T \sim 100 \mathrm{~K}$ означает, что $\lambda t \ll 10^{-12}$, где $\lambda$ - обратное время релаксации маятника, $t$ - время измерения.

\section{6. ЗАКЛЮЧЕНИЕ}

В настоящей работе мы рассмотрели частный случай стохастической формулы Бейкера-Хаусдорфа (8), в котором фигурируют две независимые компоненты винеровского процесса, причем оператор рождения входит в одну компоненту, а уничтожения - в другую. Более общий случай $n$-мерного винеровского процесса рассмотрен в работе [18].

Вычисленный в явном виде стохастический оператор эволюции $u_{t}(20)$ позволяет находить состояние системы $\rho_{t}(29)$ для произвольного начального состояния $\rho_{0}$. Формулы (31), (35) описывают поведение $\rho_{t}$ при $t \rightarrow \infty$.

Наконец, рассмотрев хорошо известную модель интерференционного метода измерения координаты подвижного зеркала, мы показали, что температура окружения измеряемой системы (осциллятора) в первом приближении по константе связи влияет только на второе слагаемое в формуле для относительных флуктуаций (45), которое не зависит от характеристик сенсора - лазерного излучения, падающего на осциллятор, и имеет смысл температурного стандартного квантового предела.

\section{Список литературы}

[1] C. W. Gardiner, P. Zoller, Quantum Noise, Springer Ser. Synergetics, Springer, Berlin, 2004.

[2] H. J. Carmichael, An Open System Approach to Quantum Optics, Springer, Berlin, 1993.

[3] V.P. Belavkin, A. Barchielli, J. Phys. A, 24:7 (1991), 1495-1514.

[4] В. П. Белавкин, ТВП, 38:4 (1993), 742-757.

[5] М. Б. Менский, Квантовые измерения и декогерениия. Модели и феноменология, Физматлит, М., 2001.

[6] V. I. Man'ko, V. A. Sharapov, E. V. Shchukin, Probability representation of kinetic equation for open quantum system, arXiv:quant-ph/0305119.

[7] M. Caponigro, S. Mancini, V. I. Man'ko, Forteschr. Phys., 54:7 (2006), 602-612.

[8] A. M. Chebotarev, Lectures on Quantum Probability, Math. Contrib. Texts, 14, SMM, México, 2000.

[9] G. Lindblad, Comm. Math. Phys., 48:2 (1976), 119-130. 
[10] S. Bose, K. Jacobs, P. L. Knight, Phys. Rev. A, 56:5 (1997), 4175-4186.

[11] C. Brif, A. Mann, J. Opt. B: Quantum Semiclass. Opt., 2:1 (2000), 53-61.

[12] А. Д. Вентцель, М.И. Фрейндлин, Флуктуации в динамических системах под действием малых случайных возмущений, Наука, М., 1979.

[13] И. В. Гирсанов, ТВП, $5: 3$ (1960), 314-330.

[14] Е. Янке, Ф. Эмде, Ф. Лёш, Специальные функиии. Формуль, графики, таблиць, Наука, М., 1977.

[15] H. A. Haus, Electromagnetic Noise and Quantum Optical Measurements, Springer, Berlin, 2000.

[16] A. M. Sinev, On a quantum model of a laser-interferometer measuring a weak classical force, arXiv: 0806.3212.

[17] V. B. Braginsky, M. L. Gorodetsky, F. Ya. Khalili, Phys. Lett. A, 246:6 (1998), 485-497.

[18] А. М. Синев, Матем. заметки, 84:3 (2008), 395-408.

Поступила в редакцию 14.07.2008 